\title{
Effect of non-drug interventions on arterial properties determined from 24-h ambulatory blood pressure measurements
}

\author{
Benjamin Gavish ${ }^{1}$, Ariela Alter ${ }^{1}$, Yael Barkai ${ }^{2}$, Carmit Rachima-Maoz ${ }^{2}$, Edna Peleg ${ }^{2}$ and Talma Rosenthal ${ }^{2}$
}

Measures derived from the slope of the linear relationship between systolic and diastolic pressures obtained by 24-h ambulatory blood pressure (ABP) measurements incorporate clinical and prognostic information, and are believed to be vascular markers. Using post hoc analysis, we investigated potential changes of these 'slope-related measures' in three different studies conducted in hypertensive patients with before and after 24-h ABP measurements, and also evaluated the sensitivity of the results to the analysis method. Two interventional studies included 8-week device-guided breathing (DGB) exercised by 13 patients with uncontrolled blood pressure (BP), and a 6-month mineral potassium chloride-enriched diet administered to 20 elderly patients. One study was observational and involved winter-to-summer change experienced by 13 patients with controlled BP. Slope-related measures included systolic-on-diastolic slope and its equivalent 1-(diastolic-on-systolic slope) called Ambulatory Arterial Stiffness Index, and were determined using three different BP-averaging methods and two types of regression procedures. Results demonstrated sensitivity of slope-related measures to the analysis method, the most significant changes were found when the before and after 24-h ABP profiles included hourly averaged BP further averaged over the patient population, and slope-related measures were determined using symmetric (and not standard) regression. DGB was found to reduce significantly all these measures. The changes in the slope-related variables for individual patients correlated negatively with its baseline value and positively with the observed pulse pressure changes. In conclusion, the study provides evidence that DGB can affect positively vascular markers associated with cardiovascular risk, and suggests improved analysis methods for the determination of slope-related measures in interventional studies.

Hypertension Research (2011) 34, 1233-1238; doi:10.1038/hr.2011.125; published online 28 July 2011

Keywords: arterial stiffness; blood pressure; device-guided breathing; lifestyle modifications; vascular health

\section{INTRODUCTION}

There is a growing evidence for an association between cardiovascular diseases and the mechanical properties of arteries and its variation with age. ${ }^{1}$ Measures derived from the slope of the linear relationship between systolic and diastolic pressures obtained by the 24-h ambulatory blood pressure (ABP) monitoring are becoming the target of research efforts, and are believed to be associated with mechanical properties of arteries. These measures, called collectively hereafter 'slope-related measures', include the systolic-on-diastolic slope $^{2}$ (S-D slope) and the Ambulatory Arterial Stiffness Index (AASI) defined by 1-(diastolic-on-systolic slope). ${ }^{3}$ Both measures were shown to be independent of mean blood pressure $(\mathrm{BP})^{2,4}$ and to incorporate clinical and prognostic information; slope-related measures were demonstrated to be independent predictors of cardiovascular and all-cause mortality; ${ }^{4,5}$ AASI predicts stroke in the general population, ${ }^{6}$ and correlates with target organ damage in hypertension. ${ }^{7,8}$ The interpretation of AASI, as a surrogate measure for arterial stiffness, ${ }^{3}$ is still debatable. ${ }^{9}$ However, a physiological model suggests that S-D slope and AASI, if calculated appropriately using symmetric type of regression procedure, ${ }^{2,4}$ express in different but equivalent ways the relative increase in arterial stiffness during the systole. ${ }^{10}$ This background suggests that chronic variations in the slope-related measures may have prognostic significance. However, we have failed to find previous studies that reported the response of these indices to interventions or a change in condition.

Slope-related measures are currently determined for an individual patient from the raw 24-h ABP data. However, the different frequency of BP reading applied during daytime and night-time is likely to bias these indices, and especially differences before and after the application of an intervention or a change in condition. In fact, averaging of 24-h ABP in constant time intervals, for example, 2-h period, was already found valuable in evaluating antihypertensive effect of drugs. ${ }^{10}$ However, the potential effect of such averaging on the slope-related

${ }^{1}$ InterCure Ltd., Lod, Israel and ${ }^{2}$ Department of Physiology and Pharmacology, Hypertension Research Unit, Sackler School of Medicine, Tel-Aviv University Medical School, Tel-Aviv, Israel

Correspondence: Dr B Gavish, InterCure Ltd., The Geophysical Institute Building, 6 Haba'al Shem-Tov Street, Northern Industrial Area, Lod 71289, Israel. E-mail: bgavish@intercure.com

Received 29 July 2010; revised 20 April 2011; accepted 19 May 2011; published online 28 July 2011 
indices in general, and its change in response to interventions or conditions, in particular, is an open question.

The study objective was to evaluate potential changes of the sloperelated measures in response to interventions or a change in condition that are known to have some antihypertensive effect. More specifically, we derived slope-related measures post hoc from three unrelated studies with hypertensive patients, ${ }^{11-13}$ in which $24-\mathrm{h}$ ABP measurements were performed before and after applying chronically nonpharmacological interventions aimed to reduce BP (two studies), and before and after a seasonal change (winter to summer). Furthermore, we investigated the effect of different methods of analyzing the 24-h $\mathrm{ABP}$ data on the results.

Part of this work has been presented as an abstract. ${ }^{14}$

\section{METHODS}

\section{Studies description}

Device-guided breathing (DGB) study ${ }^{11}$ included 8-week 15-min daily sessions in the home setting with a device that guides its user to breathe slowly with prolonged exhalation without a conscious effort (RESPeRATE, InterCure Ltd., Lod, Israel). This device is currently used for lowering high BP. ${ }^{15}$ Mineral salt diet (SALT) study ${ }^{12}$ included substitution of cooking salt with salt enriched with potassium chloride (28\%) during 6 months, which demonstrated to reduce uncontrolled $\mathrm{BP}$ at least in some of the patients (subjects were residing in an old peoples home). The seasonal change in BP (SEASON) study ${ }^{13}$ attempted to quantify the known tendency of BP to be higher during winter than summer. ${ }^{16}$ Baseline characteristics of the enrolled subjects are given in Table 1. In all studies, the antihypertensive treatment was stable between the two ABP recordings. Further details of the patients' populations are provided in the original publications. All studies have been reviewed by the appropriate ethics committees, and all patients gave their informed consent before their inclusion in the studies. It should be mentioned that these interventions were selected for evaluating the feasibility of estimating potential changes in sloperelated measures and not for the comparison, such changes by intervention, which is not justified for obvious reasons.

\section{BP measurements}

The 24-h ABP was determined in all studies using the same device, Suntech Accutracker (Suntech Medical Instruments, Raleigh, NC, USA). Frequency of BP measurements was two per hour during midnight to 0060 hours and three per hour elsewhere in both the SEASON and SALT studies and two per hour and four per hour, respectively, in the DGB study.

\section{BP analysis}

Three methods were applied for processing raw 24-h ABP data for the before and after the intervention phases: (A) Using raw data per patient: This results in

Table 1 Baseline characteristics of enrolled subjects ${ }^{a}$

\begin{tabular}{lccc}
\hline & DGB & SALT & SEASON \\
\hline Number of subjects & 13 & 20 & 32 \\
Male (\%) & 54 & 90 & 63 \\
Age range (year) & $29-70$ & $75-86$ & $20-75$ \\
Mean BMI (kg m $\left.{ }^{-2}\right)^{\mathrm{b}}$ & 25 & - & 23 \\
Taking antihypertensive drugs (\%) & $46^{\mathrm{c}}$ & $100^{\mathrm{d}}$ & $100^{\mathrm{e}}$
\end{tabular}

Abbreviations: BMI, body mass index; DGB, device-guided breathing; SALT, mineral salt diet study; SEASON, winter-to-summer change.

a Mean 24-h ambulatory blood pressure is given in Table 2 for the patients included in the analysis.

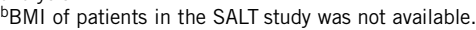

cIncluding angiotensin-converting enzyme inhibitors, $\beta$ blockers and calcium channel blockers. ${ }^{\mathrm{d}}$ The antihypertensive drugs were not specified, but diuretics were excluded. In addition, some patients received antidiuretic oral treatment and antinational and anticoagulant therapy. eIncluding diuretics, $\beta$ blockers, angiotensin-converting enzyme inhibitors, $\alpha$ blockers, angiotensin II antagonists and calcium channels blockers. a single BP datum per patient the most popular method. (B) Using hourly BP per patient: ${ }^{17} \mathrm{BP}$ is averaged in constant time intervals. We have used 1-h intervals, in the DGB and SEASON studies, and 2-h intervals in the SALT study, which generated 24,12 , and 24 BP data per patient in the DGB, SALT and SEASON studies, respectively. (C) Using hourly BP per population: The data obtained by method $\mathrm{B}$ are averaged over the entire population resulting in a single 24-h ABP profile per phase (Figure 1). The effect of the intervention on $\mathrm{BP}$ was expressed by averaging the before-to-after changes in the BP measures over the population in A); same in B after averaging over the hourly BP changes for individuals, and by averaging over the hourly BP changes in C.

Statistical evaluation of the BP changes included one-sample $t$-test. Methods $\mathrm{B}$ and $\mathrm{C}$ enable to evaluate the ratio between the mean hourly change in blood pressure (calculated over the 24 -h period), divided by the standard deviation (SD) of these hourly changes, known as the Smoothness Index that represents the dispersion of the effect of the intervention over $24 \mathrm{~h}^{18}$

\section{Slope-related measures determination}

All slope-related measures can be determined from analyzing the relationship between systolic BP (SBP) and diastolic BP (DBP) obtained by repeated BP measurements. Analysis applies either statistical software for performing regression procedures or by using analytic expressions: AASI was originally defined as 1 minus the slope $B$ in the model $D B P=A+B \times S B P$, where $A$ and $B$ are estimated using standard regression procedure. ${ }^{3}$ However, as simultaneously determined variables as SBP and DBP cannot be classified as 'dependent' and 'independent' variables, standard regression leads to artifactual dependence of AASI on the SBP-DBP correlation coefficient $r^{2}$ a more appropriate estimation can be carried out using a symmetric regression procedure of the reduced major axis typ $\mathrm{e}^{19}$ that results in $r$-independent form of AASI called hereafter 'symmetrical AASI' (AASIsym). ${ }^{2}$ Using the same method, $\mathrm{S}-\mathrm{D}$ slope can be estimated by the model $\mathrm{SBP}=\mathrm{A}+(\mathrm{S}-\mathrm{D}$ slope $) \times \mathrm{SBP}$. Software commands for this type of regression are found in literature ${ }^{18}$ and used here when the estimated error of determination is required. The 'symmetric' S-D slope can be expressed analytically by the ratio between SBP and DBP variability $\mathrm{SD}(\mathrm{SBP}) / \mathrm{SD}(\mathrm{DBP})$ called BP variability ratio (BPVR), ${ }^{4}$ where the variability is expressed by the SD of the relevant variable. ${ }^{18}$ AASIsym can be expressed by $1-1 / \mathrm{BPVR}$ and AASI by $1-r / \mathrm{BPVR},{ }^{4}$ suggesting that AASI differs from AASIsym mainly at low $r$-values. ${ }^{2,10}$ As slope-related measures are not meaningful for low $r$-values, we have excluded from analysis patients, whose $r$ was not $>0.25$ in any of the phases in the raw data when using method A

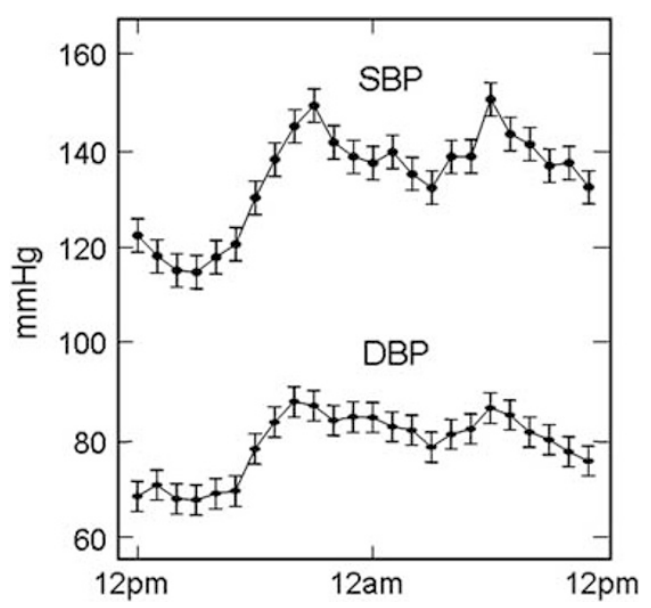

Figure 1 The 24-h ambulatory blood pressure profile generated from the raw data of the 'before' phase in the device-guided breathing study. Each point was obtained by averaging at first raw SBP (or device-guided breathing) data of an individual patient in 1-h intervals and then averaged it further over the entire population (method C). Each 'blood pressure profile' includes point obtained from the data of 13 patients. The plots and SE bars were generated using analysis of variance. DBP, diastolic blood pressure; SBP, systolic blood pressure. 
$\left(r \leqslant 0.5\right.$ has already been considered in literature as $\left.l^{2}{ }^{2}\right)$ and kept the same patients when using methods B and C. It is noteworthy that $r$ is the same for all regression methods (see Table 3).

Statistical evaluation of the change in the slope-related measures included paired $t$-test applied to values calculated for individuals (methods A and B, see Figure 3) and unpaired $t$-test after the mean and standard error (SE) were determined separately at each phase before and after the intervention from data generated by method C (Figures 1 and 2). Missing hourly averaged BP values were kept as missing to enable before-after comparison by hour. All $P$-values were two-tailed, and $P<0.05$ was selected as the minimum significance level. All calculations were carried out using the statistical software SYSTAT12.0 (Systat Software, Inc., Chicago, IL, USA).

\section{RESULTS}

Analysis included 13, 17 and 12 patients from the DGB, SALT and SEASON studies. Three patients from the SALT study and one from the SEASON study were excluded for displaying low SBP-DBP correlation in one of the phases. Only 13 24-h ABP records were available from the SEASON study. The prevalence of missing BP readings at the time interval selected for hourly averaging were $3.0,1.2$ and $3.0 \%$ of the time intervals in DGB, SALT and SEASON.
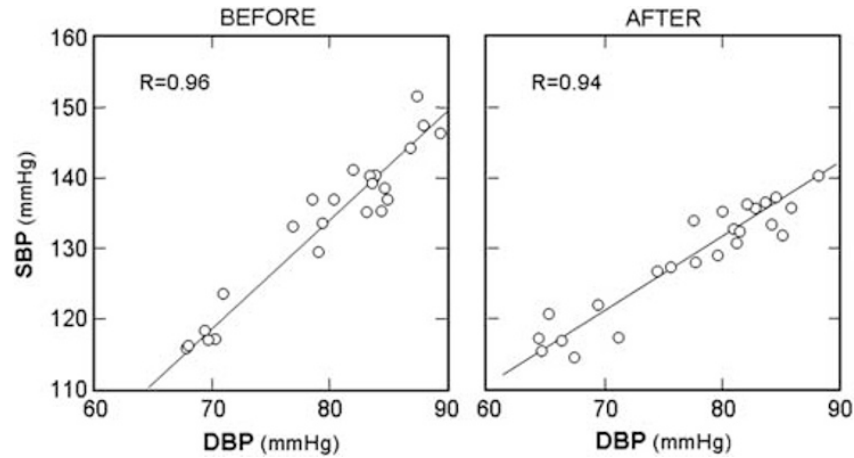

Figure 2 The relationship between SBP and DBP before and after 8-week device-guided breathing intervention constructed from the blood pressure profile shown in Figure 1 for the 'before' phase and the one corresponding the 'after' phase. The line was obtained using symmetric regression of the reduced major axis type, where the SBP-on-DBP slope was expressed by the blood pressure variability ratio and changed from $1.54 \pm 0.09$ before to $1.05 \pm 0.08$ after the intervention (mean \pm s.e., $P<0.005$ ). DBP, diastolic blood pressure; SBP, systolic blood pressure.
Table 2 shows that the different methods used for calculating BP changes in response to the interventions led to comparable results. The Smoothness Index calculated by method C appears nearly twice larger than that obtained by method B.

Table 3 shows the three slope-related measures for each intervention calculated using method $\mathrm{C}$. The changes of almost all variables appear to follow similar trend; highly significant reduction for DGB with mean/SE of 3-4; nonsignificant change in SALT and small by significant reduction in SEASON with mean/SE of nearly 0.5 for BPVR and AASI.

The slope-related measures showed sensitivity to the method of BP analysis; taking DGB as a test case, we have found that its determination from raw ambulatory data for an individual patient (method A) resulted in the following mean baseline, change (mean \pm s.e.) and its $P$-value: 1.41 and $-0.21 \pm 0.08 \quad(P=0.02)$ for BPVR; 0.48 and $-0.05 \pm 0.04(P=0.3)$ for AASI, and 0.27 and $-0.12 \pm 0.05(P=0.02)$ for AASIsym. Repeating the calculations after hourly BP averaging (method B) provided the following results: 1.45 and $-0.24 \pm 0.29$ $(P=0.01)$ for BPVR; 0.43 and $-0.09 \pm 0.18(P=0.1)$ for AASI, 0.29 and $-0.14 \pm 0.15(P=0.006)$ for AASIsym. These results are somewhat different from those obtained by method $\mathrm{C}$ shown in Table 3.

Table 3 The response of the slope-related measures to the applied interventions (mean \pm s.e.) calculated from hourly change in blood pressure averaged over the study population (method C)

\begin{tabular}{|c|c|c|c|c|c|c|c|}
\hline \multirow[b]{2}{*}{ Intervention } & \multirow[b]{2}{*}{ Variable } & \multicolumn{2}{|l|}{ Before } & \multicolumn{2}{|l|}{ After } & \multirow[b]{2}{*}{ Change } & \multirow[b]{2}{*}{ P-value } \\
\hline & & Value & $r$ & Value & r & & \\
\hline \multirow[t]{3}{*}{ DGB } & BPVR & $1.54 \pm 0.09$ & 0.96 & $1.05 \pm 0.08$ & 0.94 & $-0.48 \pm 0.13$ & $<0.005$ \\
\hline & AASI & $0.37 \pm 0.04$ & & $0.12 \pm 0.07$ & & $-0.25 \pm 0.08$ & $<0.01$ \\
\hline & AASIsym & $0.35 \pm 0.04$ & & $0.06 \pm 0.07$ & & $-0.29 \pm 0.08$ & $<0.005$ \\
\hline \multirow[t]{3}{*}{ SALT } & BPVR & $1.55 \pm 0.20$ & 0.92 & $1.56 \pm 0.24$ & 0.88 & $+0.01 \pm 0.32$ & NS \\
\hline & AASI & $0.41 \pm 0.08$ & & $0.44 \pm 0.10$ & & $+0.03 \pm 0.13$ & NS \\
\hline & AASIsym & $0.36 \pm 0.08$ & & $0.36 \pm 0.10$ & & $+0.00 \pm 0.13$ & NS \\
\hline \multirow[t]{3}{*}{ SEASON } & BPVR & $1.28 \pm 0.10$ & 0.94 & $1.20 \pm 0.12$ & 0.89 & $-0.08 \pm 0.16$ & $<0.02$ \\
\hline & AASI & $0.27 \pm 0.06$ & & $0.25 \pm 0.08$ & & $-0.01 \pm 0.11$ & NS \\
\hline & AASIsym & $0.22 \pm 0.06$ & & $0.16 \pm 0.08$ & & $-0.06 \pm 0.10$ & $<0.02$ \\
\hline
\end{tabular}

Abbreviations: AASI, Ambulatory Arterial Stiffness Index; AASIsym, symmetrical AASI; BPVR, blood pressure variability ratio; DGB, device-guided breathing; NS, nonsignificant; $r$, the systolic blood pressure-diastolic blood pressure correlation coefficient; SALT, mineral salt diet; SEASON, winter-to-summer change.

Table 2 The 24-h ambulatory BP response to the selected interventions (mean \pm s.d.) calculated in three different methods

\begin{tabular}{|c|c|c|c|c|c|c|}
\hline \multirow[b]{2}{*}{ Study } & \multirow[b]{2}{*}{ Method $^{\mathrm{a}}$} & \multicolumn{3}{|c|}{$B P(m m H g)$} & \multirow[b]{2}{*}{ P-value } & \multirow[b]{2}{*}{$S I$} \\
\hline & & Baseline & End & Change & & \\
\hline \multirow[t]{3}{*}{$\mathrm{DGB}(N=13)$} & $A$ & $136 / 81$ & $128 / 77$ & $-8.3 \pm 9.0 /-3.7 \pm 6.2$ & $0.006 / 0.05$ & - \\
\hline & B & $134 / 78$ & $127 / 76$ & $-7.4 \pm 8.3 /-3.9 \pm 6.7$ & 0.005/0.03 & $0.89 / 0.58$ \\
\hline & C & $134 / 79$ & $127 / 76$ & $-7.3 \pm 4.5 /-3.6 \pm 2.2$ & $<0.001 /<0.001$ & $1.62 / 1.64$ \\
\hline \multirow[t]{3}{*}{$\operatorname{SALT}(N=17)$} & A & $141 / 77$ & $145 / 75$ & $+3.7 \pm 20.9 . /-2.0 \pm 10.4$ & $0.47 / 0.45$ & - \\
\hline & $\mathrm{B}$ & $140 / 77$ & $145 / 75$ & $+4.8 \pm 20.8 /-1.7 \pm 9.8$ & $0.36 / 0.68$ & $0.23 / 0.17$ \\
\hline & $\mathrm{C}$ & $141 / 77$ & $145 / 75$ & $+4.8 \pm 5.8 /-1.7 \pm 3.7$ & $0.02 / 0.15$ & $0.83 / 0.43$ \\
\hline \multirow[t]{3}{*}{ SEASON $(N=12)$} & A & $131 / 77$ & $127 / 72$ & $-4.1 \pm 5.1 /-5.5 \pm 4.1$ & $0.02 / 0.001$ & - \\
\hline & $\mathrm{B}$ & $130 / 76$ & $127 / 71$ & $-3.2 \pm 4.6 /-5.1 \pm 4.0$ & $0.03 / 0.001$ & $0.70 / 1.28$ \\
\hline & $\mathrm{C}$ & $130 / 76$ & $127 / 71$ & $-3.3 \pm 5.1 /-5.0 \pm 2.8$ & $0.004 /<0.001$ & $0.65 / 1.78$ \\
\hline
\end{tabular}

Abbreviations: BP, blood pressure; DGB, device-guided breathing; method A, using raw data per patient; method B, using hourly BP per patient; method C, using hourly BP per population; $N$, number of patients used for the analysis; SALT, mineral salt diet; SEASON, winter-to-summer change; SI, Smoothness Index.

aAveraging used for evaluating BP change and P-value included $N$ for methods A and B, but the number of time intervals in method C, that is, 24 in DBG and SEASON and 12 in SALT. 


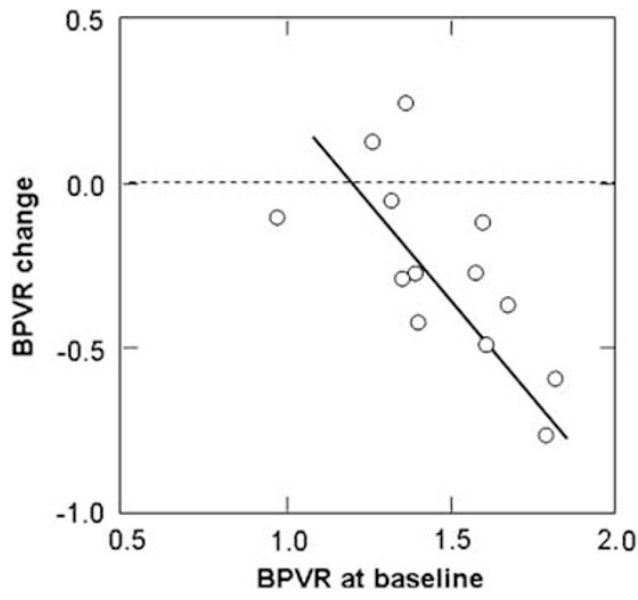

Figure 3 The dependence of blood pressure variability ratio (BPVR) change from baseline to end on its baseline value for individual patients in the device-guided breathing study $(N=13$, correlation coefficient -0.76 , $P<0.001$ for all regression coefficients). Each point was obtained from the systolic blood pressure-on-diastolic blood pressure relationship, similarly to what is shown in Figure 2, but using the raw data on the individual patients (method A). The mean \pm s.e. of baseline BPVR and change are $1.41 \pm 0.07$ and $-0.21 \pm 0.08(P=0.02)$, respectively.

The SBP-DBP correlation coefficient $r$ at the baseline phase determined from BP data calculated by methods A, B and C was $0.72,0.81$ and 0.96 , respectively.

The before-to-after changes for individuals in all slope-related measures in response to all studies negatively correlated with its baseline value. A typical example is shown in Figure 3.

Finally, slope-related measures and BP components in individuals, calculated by method $\mathrm{A}$, that displayed the highest correlated changes in response to the interventions were the followings: BPVR and pulse pressure in DGB $(r=0.73, P<0.005)$; AASIsym and pulse pressure in SEASON $(r=0.53 P=0.07)$; and AASIsym and DBP in SALT $(r=-0.65, P=0.005)$. In general, BPVR and AASIsym provided similar correlations. In contrast, AASI did not show significant or nearly significant correlation with any of the BP components.

\section{DISCUSSION}

Results demonstrate for the first time that measures related to the slope of the linear relationship between systolic and diastolic pressures obtained by 24 -h ABP monitoring can be changed by non-pharmacological interventions or conditions that are expected to affect BP. Results demonstrate similar intervention specific trend of mean change in the slope-related measures BPVR, AASI and AASIsym, reduction with DGB; no change after SALT and reduction in response to winter-to-summer transition (SEASON), where the SD of the change in the individual patients serves as a natural scale for the strength of the change (see Table 3). This finding is likely to reflect the known interdependence of these measures. Having in mind that higher value of the slope-related measures is associated with elevated cardiovascular risk or severity of condition ${ }^{4-8}$ and that slope-related measures may be expressed by arterial properties, ${ }^{10}$ the possibility of affecting these measures in the direction of risk reduction is exciting.

\section{Implications of the analysis method}

The slope-related measures are currently calculated from 24-h ABP for an individual patient using the raw data (method A), which according to the present analysis is the least sensitive method for evaluating response to the tested interventions. Using that method BPVR and AASIsym (but not AASI) still display a significant reduction in response to the DGB (Figure 3). On the other hand, the present approach of calculating the slope-related measures from the 24-h ABP data after averaging the hourly averaged BP over the population (method C, Figures 1 and 2) seems to provide greater changes and significance than the other methods. Possible reasons for that are the followings: (a) higher correlation between the systolic and diastolic BP values that gradually increases between methods in the order A, B and C, (b) larger sample value (24 data representing the hours $v s .13$ patients) and (c) smoothing individual differences between the 24-h ABP profiles before and after the intervention, as evident from the relative increase of the Smoothness Index between B and C (Table 2). All reasons reflect basic statistical properties. However, the contribution of reason $b$ to the variation between the methods depends on the size of the population in comparison with the number of the selected time intervals. Reason a explains why AASI appears similar comparable with AASIsym (and BPVR) for evaluating the intervention effect with method $\mathrm{C}$, but it looses its power of detecting changes in methods $\mathrm{A}$ and $\mathrm{B}$, this is due to the artifactual dependence of AASI on the SBP-DBP correlation coefficient $r$, which is not expressed at $r$ values close to $1 .^{2,10}$

A novel and interesting finding is the negative correlation found between the change in the slope-related measures calculated for individuals, in response to intervention, and its baseline value. This behavior was found for all measures for all the tested interventions. One possible explanation is regression to the mean-a statistical effect that appears in studies with small number of repeated measurements. $^{20}$ However, this less likely to occur when using 24-h $\mathrm{ABP},{ }^{21,22}$ suggesting that further investigation is needed for better understanding of this phenomenon.

\section{Correlates to the slope-related variable change}

The present study did not attempt to compare the response of sloperelated measures between DGB, SALT and SEASON that differ in terms of proposed mechanism of BP reduction and applied to populations with different characteristics: uncontrolled hypertensive patients in DGB, elderly hypertensive patients in SALT and hypertensive patients with controlled BP in SEASON, respectively. Instead, these interventions provided an opportunity the implications of the BP analysis methods.

As the physiological origin of the slope-related measures is still debatable and subject to investigation, ${ }^{3,9,10}$ any physiological interpretation of the results in term of mechanisms would be too speculative. An alternative approach is based on our finding that the change BPVR and AASIsym in individual patients was positively correlated with the observed change in the pulse pressure for DGB and SEASON and negatively correlated with the change in the DBP for SALT, the correlation of the slope-related measures with the pulse pressure was already demonstrated ${ }^{2}$ and interpreted by a physiological model that explains the slope-related measures as an expression for the tendency of arteries to become stiffer during the systole. ${ }^{10}$ The same model predicts positive correlation with changes in pulse pressure and negative correlation with DBP changes in the same blood vessel, as observed. The fact that DGB reduced the mean pulse pressure and SALT increased it is consistent with the predicted trend of the change in the slope-related measures. The lack of similar correlation with AASI is explained by its artifactual dependence on the SBP-DBP correlation coefficient that adds statistical 'noise' to the calculations. We may conclude that interventions or conditions that reduce the 
pulse pressure are likely to reduce the slope-related measures. In fact, wide pulse pressure has been shown to be a risk factor in middle-age and elderly persons. ${ }^{23}$

\section{Potential effects on arterial properties}

The fact that the response of the slope-related measures to SALT did not reach level of significance in the present analysis does not mean that larger sample size could not provide different results. Therefore, it may be worthwhile to summarize published evidence for physiological links between the applied interventions, modifications in mechanical properties of the brachial artery and changes in cardiovascular risk factors.

$D G B$. Slow breathing is known to reduce sympathetic activity via activation of low-threshold pulmonary stretch receptors, located in the lung and chest wall that stimulate vagal afferent fibers. ${ }^{24,25}$ In fact, a gradual change of muscle neural sympathetic activity was observed during DGB session. ${ }^{26}$ Reduction in sympathetic activity is likely to elicit smooth muscle relaxation that was demonstrated to improve brachial arterial wall mechanics in normal subjects and in patients. ${ }^{27}$ Relaxation therapy that included slow and effortless breathing has demonstrated a reduction in cardiac events in a 5-year follow-up. ${ }^{28}$ More information about the proposed mechanism for the cardiovascular effect of DGB is found elsewhere. $^{29}$

SALT. Switching from the usual salt to potassium-enriched has been shown to result in large decrease in cardiovascular disease mortality ${ }^{30}$ and to induced vasodilation of the arterial wall. ${ }^{31}$ Several clinical investigations have now firmly established the BP-independent correlations between dietary sodium and arterial stiffness, regardless of whether systemic, regional or local determinations were obtained. ${ }^{32}$

SEASON. Flow and flow-mediated dilation in the brachial artery were found to significantly associate with season, suggesting that endothelial dysfunction may be one of the mechanisms influencing the known seasonal variation in cardiovascular events. ${ }^{33}$ The suggested etiology of the increased BP in the winter is that cold increases sympathetic tone, as evidenced by elevated blood pressure and plasma and urinary noradrenaline concentrations. ${ }^{34}$

The main study limitation is the small sample size and the lack of randomized control design. However, the data included in this analysis were sufficient for evaluating the methodology of obtaining changes in slope-related measures in response to intervention or change in condition.

We may conclude that DGB has a potential for affecting positively arterial properties associated with cardiovascular risk. This study provides some indication of the direction future research should take, the potential effect of pharmacological or non-pharmacological interventions on the slope-related measures is important for exploring its effect on functional properties of arteries, especially in conditions such as pre-hypertension, where measures for vascular ageing may possess better prognostic significance than blood pressure itself. Not less important are the demonstrated sensitivity of the slope-related measures determination to the method of BP analysis and the novel type of data analysis that may optimize results.

\section{CONFLICT OF INTEREST}

Benjamin Gavish is an employee and a shareholder in InterCure Ltd., which manufactures and markets the device used in the DGB study. DGB study was sponsored by InterCure Ltd. Ariela Alter was employed by InterCure Ltd. company at the time of DGB study.
1 Lakatta EG, Levy D. Enterprises: part I: aging arteries: a 'set up' for vascular disease arterial and cardiac aging: major shareholders in cardiovascular disease. Circulation 2003; 107: 139-146.

2 Gavish B, Ben Dov IZ, Bursztyn M. Linear relationship between systolic and diastolic blood pressure monitored over $24 \mathrm{~h}$ : assessment and correlates. J Hypertens 2008; 26: 199-209.

3 Li Y, Wang JG, Dolan E, Gao PJ, Guo HF, Nawrot T, Stanton AV, Zhu DL, O'Brien E, Staessen JA. Ambulatory arterial stiffness index derived from 24-hour ambulatory blood pressure monitoring. Hypertension 2006; 47: 359-364.

4 Gavish B, Ben-Dov IZ, Kark JD, Mekler J, Bursztyn M. The association of a simple blood pressure-independent parameter derived from ambulatory blood pressure variability with short-term mortality. Hypertens Res 2009; 32: 488-495.

5 Dolan E, Thijs L, Li Y, Atkins N, McCormack P, McClory S, O'Brien E, Staessen JA, Stanton AV. Ambulatory arterial stiffness index as a predictor of cardiovascular mortality in the Dublin Outcome Study. Hypertension 2006; 47: 365-370.

6 Hansen TW, Staessen JA, Torp-Pedersen C, Rasmussen S, Li Y, Dolan E, Thijs L, Wang JG, O'Brien E, Ibsen H, Jeppesen J. Ambulatory arterial stiffness index predicts stroke in a general population. J Hypertens 2006; 24: 2247-2253.

7 Leoncini G, Ratto E, Viazzi F, Vaccaro V, Parodi A, Falqui V, Conti N, Tomolillo C, Deferrari G, Pontremoli R. Increased Ambulatory Arterial Stiffness Index is associated with target organ damage in primary hypertension. Hypertension 2006; 48: 397-403.

8 Florczak E, Januszewicz M, Januszewicz A, Prejbisz A, Kaczmarska M, Michałowska I, Kabat M, Rywik T, Rynkun D, Zieliñski T, Kuœmierczyk-Droszcz B, Pregowska-Chwała B, Kowalewski G, Hoffman P. Relationship between renal resistive index and early target organ damage in patients with never-treated essential hypertension. Blood Press 2009; 18: 55-61.

9 Schillaci G, Parati G, Pirro M, Pucci G, Mannarino MR, Sperandini L, Mannarino E. Ambulatory arterial stiffness index is not a specific marker of reduced arterial compliance. Hypertension 2007; 49: 986-991.

10 Gavish B. The relationship between systolic and diastolic pressures: a possible link between risk-related clinical measures and arterial properties. Hypertens Res 2010; 33: 657-658.

11 Rosenthal T, Alter A, Peleg E, Gavish B. Device-guided breathing exercises reduce blood pressure: ambulatory and home measurements. Am J Hypertens 2001; 14: 74-76.

12 Katz A, Rosenthal T, Maoz C, Peleg E, Zeidenstein R, Levi Y. Effect of a mineral salt diet on 24-h blood pressure monitoring in elderly hypertensive patients. J Hum Hypertens 1999; 13: 777-780.

13 Barkai Y. Seasonal variation in 24-hour ambulatory blood pressure monitoring in hypertensive patients. 2001 (MD thesis submitted to Ben-Gurion University).

14 Rosenthal T, Barkai Y, Rachima-Maoz C, Peleg E, Alter A, Gavish B. The relationship between systolic and diastolic BP: effect of season, salt and paced breathing. Am J Hypertens 2004; 17: 54A (abstract).

15 Elliott WJ, Izzo JL Jr. Nonpharmacologic treatment of hypertension by respiratory exercise. Device-guided breathing to lower blood pressure: case report and clinical overview. Med Gen Med 2006; 8: 23. http://www.medscape.com/viewarticle/539099.

16 Harvest Trial. Winnicki M, Canali C, Accurso V, Dorigatti F, Giovinazzo P, Palatini P. Relation of 24-hour ambulatory blood pressure and short-term blood pressure variability to seasonal changes in environmental temperature in stage hypertensive subjects. Results of the of the Harvest Trial. Clin Exp Hypertens 1996; 18: 995-1012.

17 de la Sierra A, Gil-Extremera B, Calvo C, Campo C, García-Puig J, Márquez E, Oliván J, Roca Cusachs A, Sanz de Castro S, Pontes C, Delgadillo J. Comparison of the antihypertensive effects of the fixed dose combination enalapril $10 \mathrm{mg} /$ nitrendipine $20 \mathrm{mg}$ vs losartan $50 \mathrm{mg} /$ hydrochlorothiazide $12.5 \mathrm{mg}$, assessed by 24-h ambulatory blood pressure monitoring, in essential hypertensive patients. J Hum Hypertens 2004; 18: 215-222.

18 Parati G, Omboni S, Rizzoni D, Agabiti-Rosei E, Mancia G. The smoothness index: a new, reproducible and clinically relevant measure of the homogeneity of the blood pressure reduction with treatment for hypertension. J Hypertens 1998; 16 : 1685-1691.

19 von Eye A, Schuster C. Regression Analysis for Social Science. San Diego: Academic Press, 1998, Chap 12, pp. 209-236.

20 Barnett AG, van der Pols JC, Dobson J. Regression to the mean: what it is and how to deal with it. Int J Epidemiol 2005; 34: 215-220.

21 Coats AJ, Radaelli A, Clark SJ, Conway J, Sleight P. The influence of ambulatory blood pressure monitoring on the design and interpretation of trials in hypertension. J Hypertens 1992; 10: 385-391.

22 O'Brien E, O'Malley K, Cox J, Stanton A. Ambulatory blood pressure monitoring in the evaluation of drug efficacy. Am Heart J 1991; 121: 999-1006.

23 Franklin SS, Khan SA, Wong ND, Larson MG, Levy D. Is pulse pressure useful in predicting risk for coronary heart Disease? The Framingham heart study. Circulation 1999; 100: 354-360.

24 Shepherd JT. The lungs as receptor sites for cardiovascular regulation. Circulation 1981; 63: 1-10.

25 Parati G, Izzo Jr JL, Gavish B. Respiration and blood pressure. In: Izzo JL, Sica D, Black HR (eds), Hypertension Primer, 4th edn. Lippincott, Williams, and Wilkins: Baltimore, 2007, pp 136-138.

26 Oneda B, Ortega KC, Gusmão JL, Araújo TG, Mion D. Sympathetic nerve activity is decreased during device-guided slow breathing. Hypertens Res 2010; 33: 708-712. 
27 Kaiser DR, Mullen K, Bank AJ. Brachial artery elastic mechanics in patients with heart failure. Hypertension 2001; 38: 1440-1445.

28 van Dixhoorn J, Duivenvoorden HJ. Effect of relaxation therapy on cardiac events after myocardial infarction: a 5-year follow-up study. J Cardiopul Rehab 1999; 19: $178-185$.

29 Gavish B. Device-guided breathing in the home setting: Technology, performance and clinical outcomes. Biol Psychol 2010; 84: 150-156.

$30 \mathrm{He} \mathrm{J,} \mathrm{Ogden} \mathrm{LG,} \mathrm{Bazzano} \mathrm{LA,} \mathrm{Vupputuri} \mathrm{S,} \mathrm{Loria} \mathrm{C,} \mathrm{Whelton} \mathrm{PK.} \mathrm{Dietary} \mathrm{sodium} \mathrm{intake}$ and incidence of congestive heart failure in overweight US men and women: first National Health and Nutrition Examination Survey Epidemiologic Follow-up Study. Arch Intern Med 2002; 162: 1619-1624.
31 Buyck JF, Blacher J, Kesse-Guyot E, Castetbon K, Galan P, Safar M, Hercberg S, Czernichow S. Differential associations of dietary sodium and potassium intake with blood pressure: a focus on pulse pressure. J Hypertens 2009; 27: 1158-1164.

32 Safar ME, Temmar M, Kakou A, Lacolley P, Thornton SN. Sodium intake and vascular stiffness in hypertension. Hypertension 2009; 54: 203-209.

33 Widlansky ME, Vita JA, Keyes MJ, Larson MG, Hamburg NM, Levy D, Mitchell GF, Osypiuk EW, Vasan RS, Benjamin EJ. Relation of season and temperature to endotheliumdependent flow-mediated vasodilation in subjects without clinical evidence of cardiovascular disease (from the Framingham Heart Study). Am J Cardiol 2007; 100: 518-523.

34 Rosenthal T. Seasonal variations in blood pressure. Am J Geriatr Cardiol 2004; 13. 274-275. 